\title{
Specification and Implementation of a Standard for Remote Database Access
}

\author{
S. Pappe, W. Lamersdorf, W. Effelsberg \\ IBM European Networking Center \\ West-Germany, Heidelberg
}

\begin{abstract}
Modern office information systems are increasingly distributed systems of heterogeneous, administrative organizational units. In general, in such systems data is held at different locations, not necessarily identical with the place at which the data is accessed and/or manipulated. There is an increasing need to access remote data without regard to computer boundaries, architectures and software specifics, thus making application programs independent of a specific database. In gencral, such communication requirements are addressed by standards efforts of ISO for 'Open Systems Interconnection" (OSI) and, in particular, by efforts of standardizing the 'Remote Database $\Lambda$ ccess'. (RDN).

Based on the current state of standardization of RDA, this paper reports on concepts and experiences of a first RDA realization. Characteristic for this implementation are the use of a formal specification method and software tools for the automatic generation of major parts of the RDA implementation.
\end{abstract}




\section{Introduction}

In office information systems or manufacturing automation, a cooperation of separate organizational units at difrerent locations is of increasing importance. In such applications, there is an increasing demand for client applications accessing centralized but remote data, without regard to computer architecture or hard- and software characteristics, via standardized vendor-independent communication interfaces. This is different from traditional, fully distributed management of data ('distributed databases') which is often difficult to achieve.

This paper is concerned with 'Remote Database Access' (RD $\Lambda$ ) in open communication networks. RDA standardizes the communication of an application program in one network node (e.g. a personal computer) with a database management system in another network node (e.g. a host computer). As both computer systems involved are, in general, of different architectural characteristics, the communication has to be based on standards as provided in the 'Open Systems Interconnection' (OSI) reference model of the 'International Organization for Standardization' (ISO) [ISO84].

The following chapter introduces the general functionality of a communication systems for RDA emphasizing the necessity for a standard RDA solution. Chapter 3 interrelates RDA with the other layers of the ISO/OSI reference model. A specific standard RDA proposal, introduced by the European Computer Manufacturers Association (ECMA), is presented in its current version in chapter 4. Topic of chapter 5 is the specification of RDA according to this proposal using a formal method. Chapter 6 discusses the implementation based on such a specification and some early experience. An outlook concludes the paper.

\section{Functionality of Remote Database Access}

In general, there are different ways to access a remote database from a personal workstation: examples include access via terminal networks, networks of autonomous nodes, distributed databases etc. [Effels87]. This paper concentrates on a case where in an open network of autonomous nodes an application program on one network node accesses a database management system on another network node. Here, the end user interacts with a local application program which then communicates with a database management system on a remote computer. In gencral, the two computers can have different characteristics. The two communication partners in RDA have different functionality: on the client site there is an application program which queries and manipulates data provided on the server site. The RDA service interfaces provides services for data query, manipulation ctc., and includes functions for conversation structuring, recovery and commitment control. So, RDA realizes a specific form of inter-process communication.

The RDA client requests are transmitted via the lower layers of open communication system to the server site where they are presented to the server process. This server process is responsible for translating the requests/responses to and from the database management system. The client communicates with the database server exclusively based on RDA primitives. So, the RDA primitives mask the specific characteristics of the database from the database client as wcll as, vice versa, the RDA also masks the client from the database. This allows the application program and the database management system to be developed independently and still be able to communicate in an open systems environment. 
Besides the service interface, the communication standards include the detailed communication protocol and the mappings to the lower layer services.

Figure 1 gives an overview of the functionality of RDA in an open systems environment.

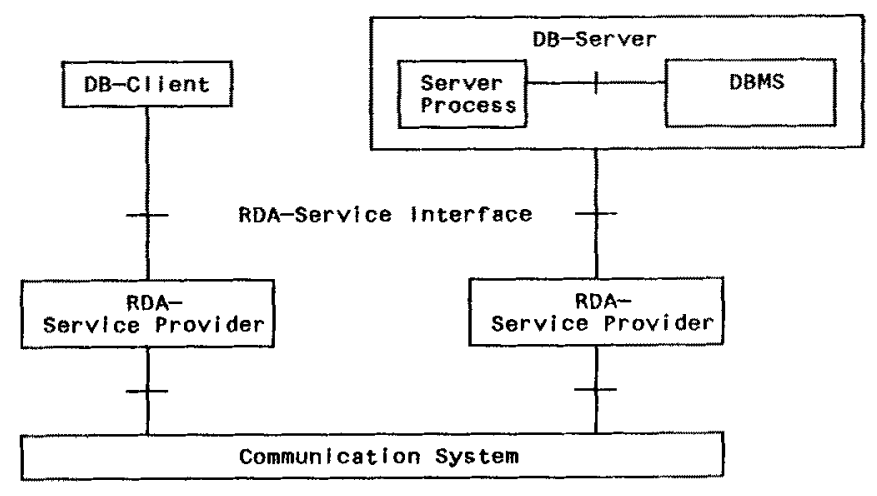

Figure 1. Remote Database Access in Open Systems

As a full OSI RDA implementation also involves the lower OSI layers, the following chapter briefly reviews the OSI model and concentrates on embedding RDA into it.

\section{Imbedding RDA into the OSI Reference Model}

The ISO reference model (standardized in 1984 in its present form) is subdivided into the well-known seven layers which can be grouped into two main parts: the transport-oriented layers 1 to 4 depend mainly on the characteristics of the underlying media, and the application-oriented layers 5 to 7 are mainly oriented towards the application problem to be solved. [Gicse85,Goergen85, Effels86]. Standardization of the seven layers is a slow process: Currently, layers 1 to 3 are provided by many PTTs in packet switching networks (e.g., Datex-P in Germany); layers 4 (transport) and 5 (session) are now international standards, and layer 6 (presentation) is a Draft International Standard (DIS). Layer 7 (application) is still an area of active research. Current efforts are going in two directions: the monolithic definition of specific application protocols (e.g., X.400 for message handling), and the standardization of a sub-structure within the application layer. One proposal is to subdivide layer 7 into a 'lower' part which provides the more generic functionality for several applications (Common Application Service Elements, CASE), and an 'upper' part which defines specific end user applications ([ECMA85, ISO86a,ISO86b,ISO86c,ISO86d,ISO86g]). Examples for such specific applications include file transfer (FTAM), remote database access (RDA) and others. Figure 2 shows the position of RDA in the OSI reference model, including common application service elements. 


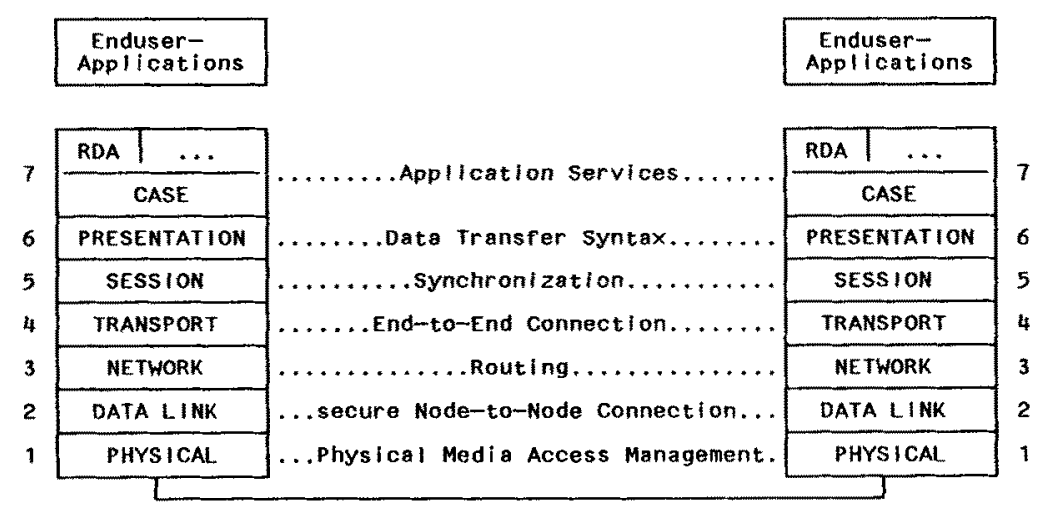

Figure 2. ROA in the ISO/OSI Reference Model

One of the major problems to be solved in RDA is the mapping of the RDA protocol to underlying common application service elements within layer 7. The following chapter reports on an RDA standard proposal describing one approach to this problem.

\section{The ECMA RDA Standard Proposal}

Since 1985, Technical Committee 22 (on Databases) of the European Computer Manufacturers Association (ECMA) is working on the standardization of remote database access. The latest version of the ECMA standard proposal [ECMA86] has, in the meantime, been introduced to ISO. This proposal includes more recent developments within ISO with respect to the architccture of the application layer.

In the ECMA proposal, the services offered by RDA enable a database client to access and manipulate remote databases, and a server database process to react to such requests. $\Lambda$ ccording to that proposal, the RDA services can be classified into four different groups [Pappe87]:

- Connection /Association Management

- Resource Management

- Data Query and Manipulation

- Transaction Management.

An Association is a communication relationship between a databasc clicnt and the database server which can - other than in lower layers - not be discontinued without the permission of the two communication partners. In case a connection has to be disrupted by the lower layers, the association still exists and can be revived whenever a new connection is established.

Resource Management is concerned, on one hand, with access to the resources of the database (i.e., depending on granularity, access to the whole database, a relation or a single record). On the other hand, it also includes management of information about the databasc (depending on its type). 
An RDA Transaction is a logical unit of work with "all-or-nothing" property. Each client is allowed to have one open transaction per connection at each point in time. The database server can have more than one open transaction if it has several concurrent users.

Services for Data Query and Manipulation, enable the client to request database action to either retrieve or to manipulate data. The server responds to such requests either with a result (resp. status) or with an error message.

The interrelationship of all RDA services is shown in the RDA protocol automaton, see Figure 3.

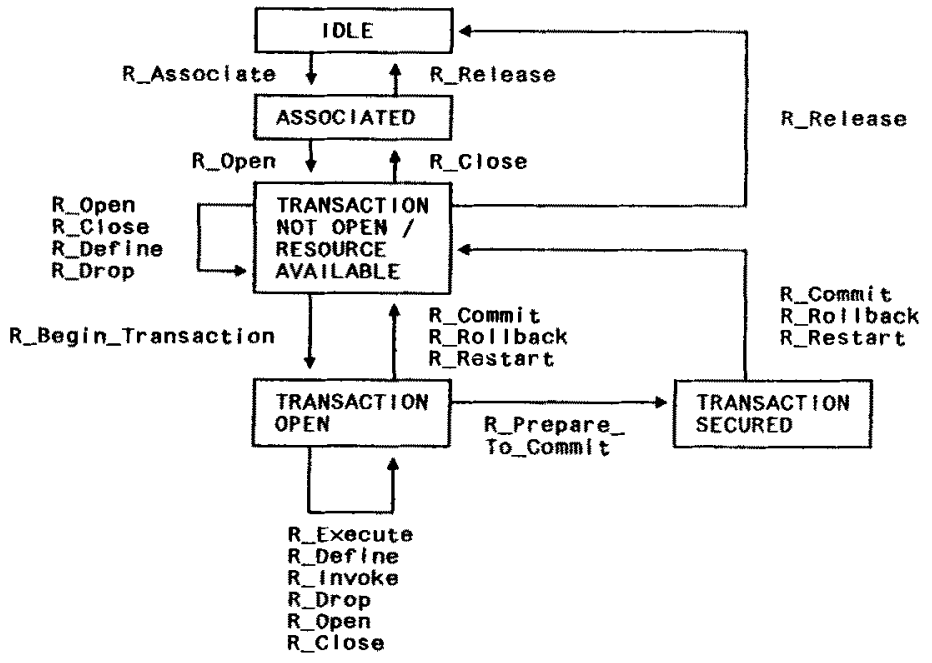

Figure 3. RDA protocol automaton according to the second ECM $\Lambda$ proposal

The RDA protocol automaton has five states. From the initial state IDI.E, R- $\Lambda$ SSOCIATE establishes an association between client and server, and the following state is $\Lambda$ SSOCIATED. Here, access to database resources can be gained. Using R-RELEASE, an association is closed, leading back to the initial state IDLE.

Acquiring a first resource leads to the state TRANSACTION NOT OPEN / RESOURCE AVAILABLE. Using the service R-OPEN, additional resources can be opened or released or locked. The services R-DEFINE and R-DROP allow for storage and deletion of stored database commands. Stored database commands can be executed later based on a returned identification.

A transaction is opened with the R-BEGIN-TRANSACTION service. This leads to the state TRANSACTION OPEN, where requests for database management statements are possible. These are invoked one by one using the service R-EXECUTE. Also, in this state, database management statements or sequences of such statements can be stored, executed, and deleted, and additional resources can be opened or closed. 
A transaction is closed by either a 1-phase commit or a 2-phase commit (via state TRANSACTION SECURED). Using the service R-ROLLBACK, a running transaction can be rolled back; using R-RESTART it is possible to resynchronize the client and the server after a failure.

Besides specifying the parameters of the above mentioned services, there is a need for uniquely representing the variable values in database commands as, e.g, actual request parameters or database query results (data representation). A simple way to transmit these values is to send them as strings. There are, however, problems using this approach: In an open systems environment, one has to deal with different, machine-dependent character representations and language dialects. As RDA is based on a standardized database query and manipulation language (syntactically defined in the RDA document in ASN.1, the ISO specification language for data types and data structures [ISO86e]), database language statements to be sent can already be parsed at the client's site and then be transmitted in an intermediate tree representation, as uniquely defined by the ASN.1 specification. In this way, incorrect statements can be rejected already at the client's site, in order to minimize communication cost.

The ECMA RDA standard proposal is based on the above approach. The standardized database language SQL is defined in ASN.1 in the proposal. Encoding for transmission is done according to the 'Basic Encoding Rules for ASN.1' [ISO86f]. The use of SQL represents only one of several possibilities of database languages; the RDA proposal distinguishes between a part that depends on the data model and data manipulation language and a generic part. The problem of transmitting variable data (i.e. parameters and results) is solved by defining - in RDA - a unique encoding of such values which includes their own description.

The protocol specification of RDA defines the correct contents of the protocol data units (PDUs) to be exchanged and specifies the expected behaviour of the peer instances over time. The asymmetry of the two communication partners (client and server) is mirrored by two different protocol automata. One important aspect to be defined by the protocol automata is the reaction to errors. In addition, the protocol specification describes how to map the RDA protocol to the lower ISO/OSI layers and/or common application service elements. The latest versions of the RDA standard are characterized by not basing RDA directly on the presentation layer (6), but rather using some of the common application service elements provided by a 'lower' part of the application layer (7).

Using common application services, a major part of an application's functionality can be moved to lower service elements where it can also be useful for other applications as well. This implies that the RDA protocol automaton is reduced substantially in complexity. Lxamples for functionality to be moved down are remote operation calls, association management and commitment control.

What is currently missing in the OSI application layer model, however, is a conceptually clear distinction between common and specific application service elements and their interrelationship. If more than one common applications service element is used, not only the relationship to the user (here: RDA) and its communication partner but also the relationship to other common application service elements has to be clearly defined. At the time being, each end user application (e.g. RDA) defines its own relationship of subcomponents to be used and no generally accepted framework for substructuring the application layer exists. A first proposal is under development at IBM's European Networking Center (ENC) in Heidelberg [Bever87]. 
In conclusion, the major characteristics of the ECMA RDA proposal can be summarized as follows:

- The ECMA proposal is based on common application service elcments, i.e. the RDA services are not mapped directly to the presentation layer. This simplifies the RDA protocol considerably.

- The ECMA proposal is not restricted to a specific database language, i.e. the RDA service definition is given in a very general framework with possible specializations for alternative database languages. In general, such a use of more generic scrvices for a more specific purpose is called specialization. The actual language to be used is defined during association establishment. An annex to the RDA specification presents an SQL specification as a specialization for one possible database language.

After presenting first the general requirements for remote database access in an OSI environment and then a current proposal for a specific RDA service element, the following chapters concentrate on implementing such a proposal in a real system environment. The implementation is based on a formal protocol specification language and a set of related tools which have alrcady proved to be useful for implementing other ISO/OSI standards proposals [Fleisch87a].

\section{Formal Specification of $R D A$}

As a first step, and as a prerequisite to automatic code generation, RDA is specified formally with the specification technique PASS (Parallel Activity Specification Scheme). PASS is a method for specifying a complex system in the form of communicating parallel processes, e.g., in process control applications or communication systems [Andres84a, Andres84b, Fleisch87a, Fleisch87b]. The technique was originally developed at the University of Erlangen-Nürnberg and extended at IBM's European Networking Center. Practical experience with PASS was gained in the implementation of the ISO/OSI layers 4, 5 and 6 (Transport, Session and Presentation) [Frantz86, Pappe86, Fleisch87a].

\subsection{Introduction to PASS, the Parallel Activity Specification Scheme}

A PASS process description is based on the extended finite state machine model. Finite state machines are extended with variables for storing information; these additional variables are a prerequisite for the convenient description of complex communicating processes. In the context of OSI sofware, a PASS specification is an intermediate step between the standard document (c.g., from ISO, ECMA or CCITT) and the code in a programming language. Unlike the standard documcnt, a PASS specification is fully formal. It resolves all imprecisions and ambiguities in the standard which are due to the fact that a standard cannot take the properties of the implementation environment into account (hardware, operating system, programming language, etc.). A PASS specification is detailed enough to derive programming language code (e.g, in Pascal) automatically. The role of PASS in the software development process is shown in Figure 4. 


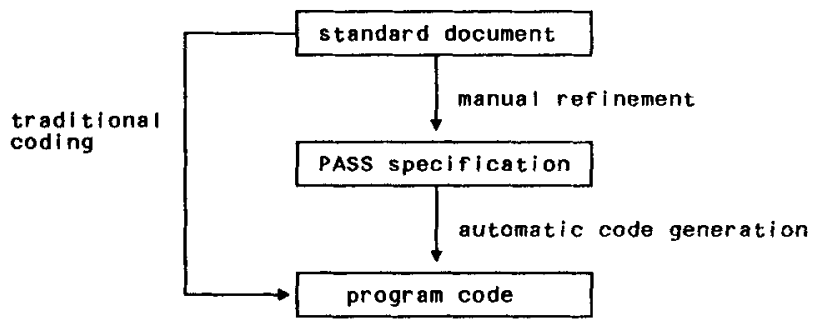

Figure 4. PASS in the OSI software development process

In PASS, a distributed system consists of processes communicating via messages. Each process has a unique name. The number of processes in a system is static. $\Lambda$ messagc consists of a message name and message parameters.

A process description has two major parts:

- a PASS graph

- PASS graph refinements.

The PASS graph describes the allowable sequences of transitions in a process. The graph consists of nodes and directed edges, corresponding to the states and transitions of the finite state machine. There are four types of nodes; two types of communication nodes and two types of internal nodes, as follows:

A send node describes a state in which a process wants to send a message to another process. This message can be sent synchronously or asynchronously. For asynchronous communication, PASS provides the concept of input pools (buffers). If an input pool has size 0 , the message exchange is synchronous, i.e. there is a rendezvous between the sending and the receiving process. $A$ send node can have one or more outgoing edges. Each edge is marked with the name of the message and its destination process. When a message is sent, the corresponding transition is made in the graph. If more than one message can be sent at a time, prioritics can be assigned. In order to avoid infinite blocking of a process, a time-out transition can be specified. The timc-out transition is executed when none of the messages can be sent betore time-out (no recciver ready, or all receiver input pools full).

A receive node corresponds to a state where a process expects a message from another process. If a process has an input pool and the expected message is in the input pool, the transition to the next state is made. If a process has no input pool but the expected message is offered by the sending process synchronously, the transition to the successor state can also be performed. If the expected message is not in the input pool (receiving process with input pool) or is not offered by the sending process (receiving process without input pool), the receiving process is blocked. Receiving of different messages, possibly from different processes, is allowed in one receive state; a corresponding number of edges leaves the state. Each edge is marked with the message type and the name of the sending process from which the message is expected. A priority list can be defined for receive nodes 
with more than one edge, just like for send nodes. Infinitc blocking can also be avoided with a time-out edge.

An internal function node corresponds to a main state where a process evaluates local variables. Since PASS is based on extended finite state machines, it must be possible to evaluate the status of local variables and make transitions based on their values. The computations of an internal function are deterministic; therefore, there are no prioritics, and thcre is no time-out. Internal functions do not modify local variables.

An internal operation node corresponds to a state where a process assigns new valucs to local variables. Depending on the computed new values, different transitions to successor states can be made. For example, a successful execution of an operation can lead to one transition, an unsuccessful execution to another. Like an internal function, an internal operation is deterministic; there are no priorities and there is no time-out.

The pictorial representation of a PASS graph uses boxes and ovals for the states and single-lined and double-lined arrows for the transitions, as shown in Figure 5.

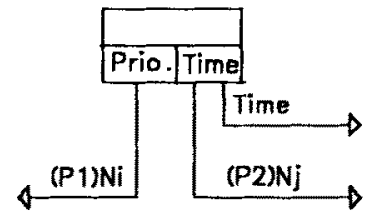

Receive node

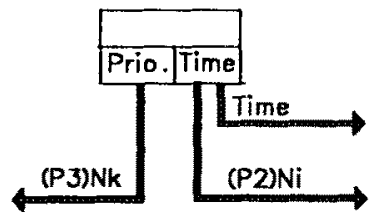

Send node

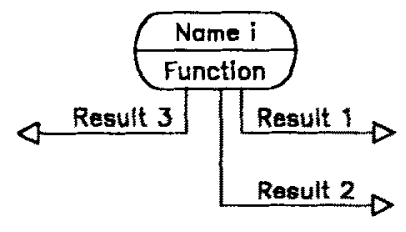

Function node

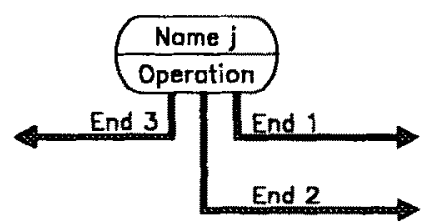

Operation node

Figure 5. The four node types of PASS

A complete PASS diagram (a PASS graph) describes the state-transition bchavior of a process and all of its external interactions, i.e. all the messages it will send or reccive. In addition to specifying the communication behavior, it is necessary to describe the internal functions and operations in some kind of formal language. This part of a PASS specification is called PASS graph refinements. Since internal functions and operations are purely sequential, any formal language for sequential processes can be used. In the current version of PASS, the graph refinements are specified in Pascal. Pascal was chosen for two reasons: the language is very widely used and thus familiar to many people. In addition, the 
current version of the code generator generates Pascal code for the PASS graph part; it is thus straightforward to integrate the PASS graph refinements with the code generated for the PASS graph.

So far, we have described the specification technique PASS in gencral. In principle, PASS can be used for any system of communicating processes. In the following section, we explain the use of PASS for layered communication protocols.

\subsection{Specification of Communication Protocols with PASS}

In a system of layered communication protocols, each layer has its own protocol to communicate with its peer entity. It uses the services of the next lower layer for this purpose. These services are offered in the form of service primitives.

In the ISO reference model, the concept of Service Access Points (SAPs) was introduced. One entity can have several service access points, each with a different service access point address; but each service access point is uniquely assigned to a single entity. If a layer implements multiplexing, several service access points can be mapped to one service access point of the next lower layer. The mapping of 'upper' service access points to 'lower' service access points is one of the tasks of a layer. For layer $\mathrm{N}$ in a hierarchy, this is called ' $\mathrm{N}$-mapping'.

In PASS, each protocol entity is described as a process. Each service primitive at a service interface is described as a message. The N-mapping is done by a special process, the Layer Manager. Since a layer can contain several protocol entities at the same time (i.e., when multiple parallel connections are active), the Layer Manager also schedules the protocol entities within a layer. The Layer Manager receives the incoming messages, including the service request messages from the layer above and the indication messages from the layer below, and forwards them to the appropriate protocol entity. When a connect request or a connect indication arrives, and thus a new connection must be established, the Layer Manager creates a new instance of a protocol entity and enters the connection identifier of the new connection into the $\mathrm{N}$-mapping table. Thus the protocol entitics themselves do not have to maintain the N-mapping information. Each of them implements just the layer protocol machine.

Figure 6shows the specification of layered protocols with PASS, and in particular the relationship of protocol entities to their layer managers. 


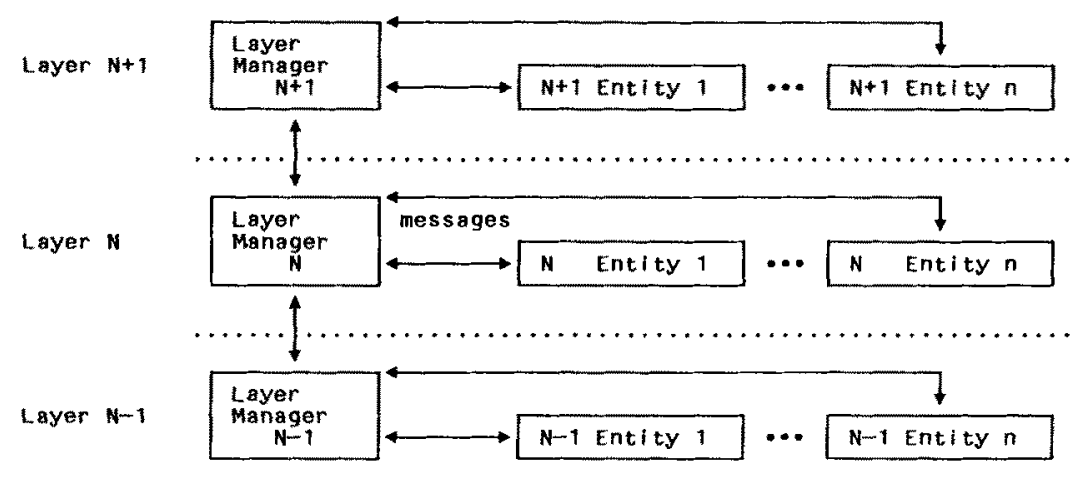

Figure 6. PASS processes implementing a layered communication system

First experience with PASS has shown that the technique was very valuable [Frantz86, Pappe86, Fleisch87a]:

- it is easy to learn and use,

- code can be produced automatically from a PASS specification,

- use of the PASS methodology forces the implementor to a well-structured design, and

- the layer code produced with the PASS code generator is well-structured and easy to debug.

Therefore, it was decided to use PASS again for the specification and implementation of RDA.

\subsection{Application of PASS to Remote Database Access}

From the previous two sections, it is now clear that a PASS description of RDA will have the following components:

- a PASS Graph for the RDA protocol entity,

- PASS Graph Refinements for the RDA entity, and

- an RDA Layer Manager.

The RDA PASS Graph describes the allowable sequences of messages sent and received by the RDA protocol entity, and the internal functions and operations performed. The RDA PASS Graph Refinements define the local variables (e.g., control blocks) and the exact details of each of the nodes in the PASS Graph. The Layer Manager performs the N-mapping (i.e., the management of SAPs and the correct distribution of messages to the protocol entities within the layer).

The specification of the PASS graph follows closely the ECMA standard proposal. In the current version, the protocol automaton is only defined very schematically (see Figure 3 ). There are no hints for the introduction of local variables and their use in the protocol. Such information is usually quite helpful to describe the detection and handling of errors. Two classes of errors can be distinguished: the invocation of services out of sequence (illega! events), and illegal values for parameters in service primitives (illegal parameters). Illegal events are easy to handle: the protocol machine code detects an 
illegal event (in the current state, there is no provision for an event of this type). Illegal parameters are more difficult to handle in RDA. As shown in section 2, the server site of RDA includes the database management system. Since the RDA standard must be independent of any specific DBMS, no assumptions can be made about the error detection capabilities of the DBMS for illegal parameter values coming through RDA. Thus the exact error handling behavior of the server site is not clear, and cannot be specified as part of the standard.

For RDA, the PASS Graph Refinements were specified in Pascal. They consist of the specification of the internal functions and operations, where most of the code goes into crror handling, and of the details of the send and receive operations (in particular, the computation of local variables from receive message parameters and the computation of send message parameters from local variables).

In our first prototype, the specification of the Layer Manager process was kept simple in our first prototype. No mapping to a real lower OSI layer or CASE service clements was done. Instead, simulated OSI services were used as a transport mechanism. The routing of messages to RDA protocol entities on the server site is based on userids under the VM operating system. In a later version of the prototype, the $\mathrm{N}$-mapping will implemented as part of the Layer Manager.

Once a complete PASS specification of the Remote Database Access layer was available, it had to be translated into programming language code. As mentioned earlier, we decided to use Pascal as our programming language. In the following, we describe the generation of Pascal code from the PASS specification. We also describe the tools used in the specification and code generation process, and early test experience.

\section{Implementation and Experience}

\subsection{Implementation of RDA}

The specification and implementation process with PASS is supported by two software tools: a PASS graph editor and a PASS code generator. Both tools have uscr-friendly menu-driven interfaces.

The interactive specification of a PASS Graph is supported by the PASS Graph editor. In the current version, there is no graphical interface; instead, an equivalent line-oriented notation is used. This notation is more convenient for large, complex protocol specifications where the PASS Graph is too large to fit on a screen. One line represents one transition, defined by the starting state, the successor state and the triggering event. As an example, Figure 7 shows a PASS receive state in the graphical and the equivalent line-oriented notation. 
GRAPHICAL. REPRESENTATION

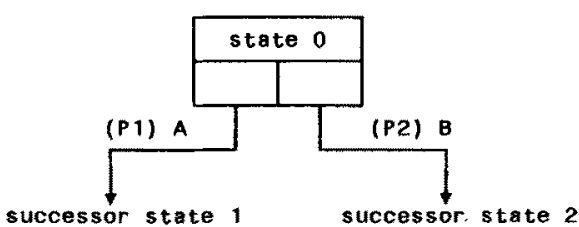

LINE-ORIENTED NOTATION

(state 0$) \longrightarrow(R)(P 1) A \longrightarrow$ (successor state 1)

(state 0$)-(R)(P 2) B \rightarrow($ successor state 2 )

$\lfloor R$ means receive event

Figure 7. Graphical and line-oriented notation of the PASS Graph

In addition to the editing function, the PASS Graph editor supports graph-theoretical plausibility checks to guarantee a syntactically correct PASS graph. For example, nodes with no outgoing edges (sinks) or nodes with no incoming edges (sources) are detected. The designer can then correct the PASS Graph without leaving the editor. These plausibility checks operate on the static graph only; They should not be confused with protocol validation [West86]. The PASS Graph editor also includes a graph optimization algorithm. It detects and removes multiple identical transition sequences (e.g., in an error-handling part of the protocol), saving about $10-20 \%$ of the PASS states in typical protocols.

The specification of the PASS graph refinements is done by hand in Pascal, as mentioned earlier. First, the designer speciffes the data types and variables on which the operations and functions operate. From these data declarations and the PASS Graph, procedure headers for all internal functions and operations will be generated. Only the procedure bodies are coded by hand.

The second tool is the PASS code generator. Input of the generator are the PASS Graph, as created by the editor, and the data types and variables of the refinements. Out of this the generator produces the complete code for the PASS graph and the code frames (procedure headers) for the PASS graph refinements. Combining these code frames with the manually coded procedure bodies leads to the complete code for the protocol entity. An overview of the design and implementation procedure is shown in Figure 8. 


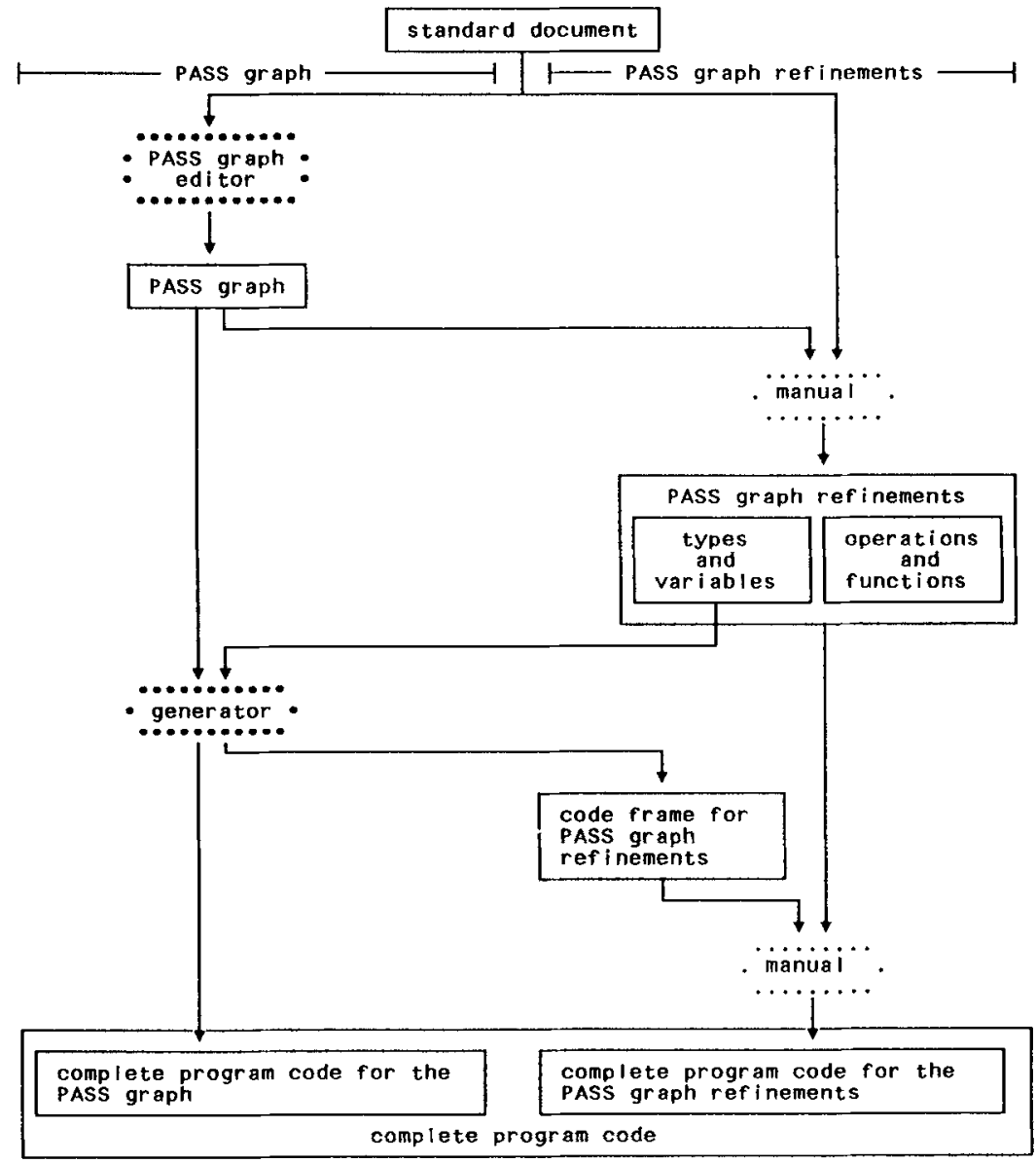

Figure 8. Implementation of OSI Software with PASS and PASS Tools

The implementation of the Layer Manager is facilitated by an "Open Systems Environment (OSE)", an operating system enhancement for OSI, as described in chapter 6.3.

\subsection{Status of the RDA Prototype}

Our first RDA prototype consists of an RDA client and an RDA server as two separate processes. With a given transport system these processes can also be physically scparated. The client has no protocol machine, the server a complete one. Incorrect service requests (illegal events or illegal parameters) are only detected by the server. This implies additional communication overhead in case of faulty requests but simplifies the client process considerably. 
In the current prototype, two-phase commit is not implemented because the underlying DBMS does not support it.

For data query and manipulation commands the prototype supports (as a specialization of the generic facility) the database language SQL. SQL keywords as well as arguments (parameters) and results are 'privately' encoded, i.e. the way of encoding is in the responsibility of the communication partners. The use of ISO presentation layer services for this purpose is under investigation.

The mapping of the RDA protocol to lower CASE services is actually only emulated because those software components are still under development and because the mapping onto those services is currently in a intensive discussion phase (i.e., not yet stable). Up to now, the C $\triangle S E$ requests are transmitted via a non-OSI operating system communication facility.

The presented prototype was developed under the operating system VM/SP on an IBM 3083 computer. For portability reasons, the programming language Pascal was chosen. The RD $\Lambda$ server is based on the SQL/DS database management system. A Pascal interface to SQL/DS had to be built in order to get access to SQL/DS from Pascal.

\subsection{Imbedding the RDA Implementation into an Operating System Environment}

The purpose of OSI software is the interconnection of heterogeneous computers. There is an inherent interest in writing it in a portable fashion so that identical layer implementations can run on systems with different architectures (hardware and operating system). On the other hand, OSI software is system software; it needs direct access to system resources, such as message buffers, timers, an operator console, etc. How is it possible to write portable system software?

One approach to solving this contradiction is an Open System Environment (OSE), providing operating system services to OSI layers through a standardized interface. Instcad of re-writing all OSI layers, only the implementation environment is re-written for each new machine [Flcisch87a]. The OSI layers use operating system services only through the OSE. The real operating systems can differ on various computers, but the OSI sofware is identical (see Figure 9). This presumes that the OSI layers are written in a portable language.

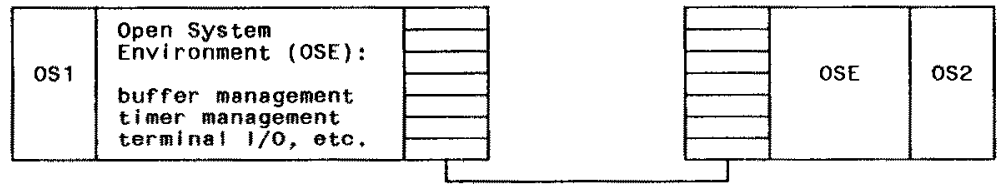

Figure 9. An Open Systems Environment for portahle OSI software 


\subsection{Testing}

Besides specification and implementation of RD $\Lambda$, testing of the RD $\Lambda$ software (and of OSI software in general) is an interesting problem. The OSI software must conform with the standard. There are two different kinds of conformance requirements to be fulfilled: static conformance and dynamic conformance. Static conformance determines which capabilities of the RDA standard proposal are supported by the current implementation. Dynamic conformance is reached if the cxternal behavior of an implementation conforms to the standard.

Because of the current restrictions of the prototype, the dynamic behavior can be tested only incompletely. Statically the supported functions were tested with the tools explained in the following.

The actual implementation consists of four main parts:

- $\operatorname{RDA}$ (client role)

- $\operatorname{RDA}$ (server role)

- RDA-database interface

- CASE simulation.

Figure 10 shows the test environment. $\wedge$ common user-friendly testing interface (UFT) is provided for testing different configurations. This interface provides a comfortabic fullscreen $1 / O$ facility with the option of saving test scenarios or executing saved test scenarios.

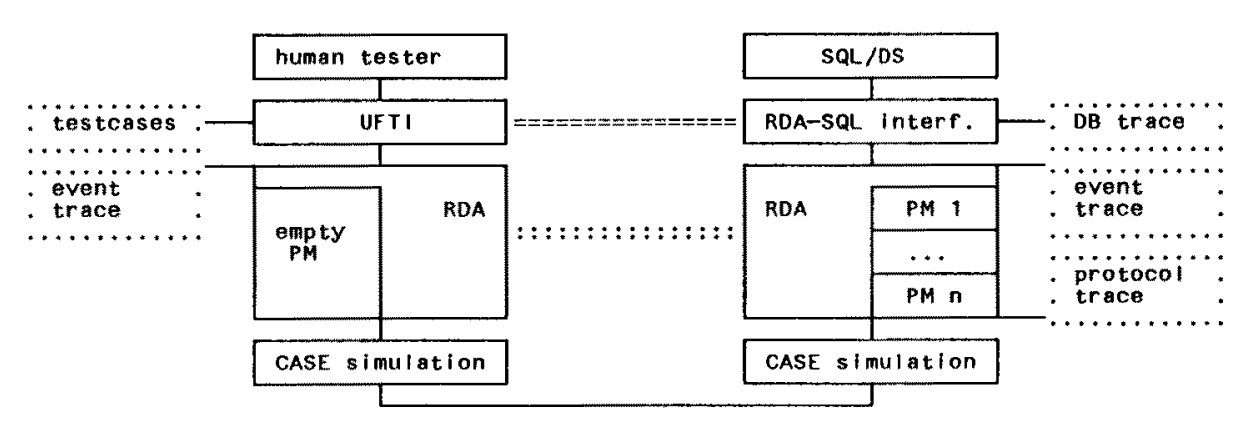

Figure 10. Test configuration

Testing of the complete implementation is done in the following way: with the help of UFTI the user requests services from the client RDA. These are traced on a filc. Because the client RDA has no protocol machine the requested services are directly passed on to the CASE simulation which is responsible for the transport to the server site. A new association at the server site causes the creation of a new instance of a protocol entity, as described in Chapter 5.2. Subsequent requests on the same association are routed to the correct protocol entity. The CASE and RDA-SQL/DS interface events are traced. If the requests are accepted by the RDA server entity, they are delivered to the RDA-SQL/DS interface which translates them into SQL/DS calls. These calls are also traced. 
In this way the cooperation of all components is tested. For the testing of subsystems, UFTI provides the facility to access directly the RDA-SQL/DS interface $(===$ in Figure 10) or to test the server locally, without CASE simulation (::: in Figure 10). All three test configurations are accessed through a uniform surface.

\section{Outlook}

In the near future the RDA prototype will be embedded into an OSE, as described above. In parallel, prototypes of common application service elements are being developed and integrated under a new upper layer architecture.

In addition to the mapping of RDA onto common application service elements proposed by ECMA, other mappings onto different service providers are thinkable. The influences of this on the RDA functionality will be the subject of our future work.

In the long term, an extension of RDA to include support for complex objects of multi-media databases (including voice, images, video, etc.) must be investigated. Also, with the arrival of high-speed networks with data rates above $100 \mathrm{Mbit} / \mathrm{s}$, the performance of the RDA protocol and its mappings to lower layers must be evaluated.

\section{Acknowledgments}

We would like to thank our colleagues at the ENC for many intensive and helpful discussions. In particular, Albert Fleischmann, the inventor of PASS, and Martin Bever, our expert on upper layer arehitecture and CASE, helped us very much. We also thank the colleagues in ECMA TC 22 for many good and open technical meetings.

\section{References}

[Andres84a] C. Andres, A. Fleischmann, P. Holleczek, U. Hlilmer, R. Kummer: Eine Methode zur Beschreibung von verteilten Programmen, angewandt bei der Spezifikation von DFÜ-Protokollen. Regionales Rechenzentrum der Universität Erlangen-Nürnberg, 1984.

[Andres84b] C. Andres, A. Fleischmann, P. Holleczek, U. Hillmer, R. Kummer: Die Umsetzung einer Spezifikationsmethode für DFÜ-Systeme in verschiedene Arten von Programmiersprachen. Regionales Rechenzentrum der Universität Erlangen-Nürnberg, 1984.

[Bever87] M. Bever, A. Fleischmann: Ein Konfigurationskonzept für die Anwendungsebene des ISO-Referenzmodells für offene (Buro-)Systeme. To appear in Proc. GI Annual Conference, Munich, 1987.

[Ceri84] St. Ceri, G. Pelagatti: Distributed Databases - Principles and Systems. Mc Graw-Hill, 1984.

[Eckh86] H. Eckhardt, W. Lamersdorf, K. Reinhardt, J.W. Schmidt: Datenbankprogrammierung in Rechnernetzen. GI-Jahrestagung, Berlin, Springer Verlag, 1986.

[ECMA85] ECMA: Remote Operations - Concepts, Notation and Connection-Oriented Mappings. ECMA Technical Report 31, December 1985.

[ECMA86] ECMA: Remote Database Access. Second Working Draft for a Standard, December 1986. 
[Effels86] W. Effelsberg, A. Fleischmann: Das ISO-Referenzmodell für Offene Systeme und seine sieben Schichten. Informatik Spektrum, Bd. 9, Heft 5, October 1986, pp. 280-299.

[Effels87] W. Effelsberg: Datenbankzugriff in Rechnernetzen, in: it (Informationstechnik), 29. Jahrgang, Heft 3 (1987), pp. 140-153.

[Fleisch87a] A. Fleischmann, S.T. Chin, W. Effelsberg: Specification and Implementation of an ISO Session Layer. 'To appear in IBM Systems Journal, 1987.

[Fleisch87b] A. Fleischmann: PASS-A Technique for Specifying Communication Protocols. Proc. Protocol Specification, Testing and Verification VII, Zurich, North Holland, 1987.

[Frantz86] K. Frantz: Generierung von Protokollen aus einer PASS-Spezifikation am Beispiel der ISO/OSI-Transportebene. Diplomarbeit, Fachbereich Informatik der Univ. Frankfurt, 1986.

[Giese85] E. Giese, K. Goergen, E. Hinsch, G. Schulze, K. Truoel: Dienste und Protokolle in Kommunikationssystemen. Springer Verlag, 1985.

[Goergen85] G. Goergen, H. Koch, G. Schulze, B. Struif, K. Truocl: Grundlagen der Kommunikationstechnologie; ISO-Architektur offener Kommunikationssysteme. Springer Verlag, 1985.

[ISO84] ISO: International Standard 7498. Open Systems Interconnection - Basic Reference Model, 1984.

[ISO86a] ISO: International Standard 8649/2. Open Systems Interconnection - Service Definition for Common Application Service Elements - Part 2: Association Control, 1986.

[ISO86b] ISO: International Standard 8650/2. Open Systems Interconnection - Protocol Specification for Common Application Service Elements - Part 2: Association Control, 1986.

[ISO86c] ISO: International Standard 8649/3. Open Systems Interconnection - Definition of Common Application Service Elements - Part 3: Commitment, Concurrency and Recovery, 1986 .

[ISO86d] ISO: International Standard 8650/3. Open Systems Interconnection - Specification of Protocols for Common Application Service Elements - Part 3: Commitment, Concurrency and Recovery, 1986.

[ISO86e] ISO DIS 8824; Specification of Abstract Syntax Notation One (ASN.1), May 1986.

[ISO86f] ISO DIS 8825: Basic Encoding Rules for Abstract Syntax Notation One, 1986.

[ISO86g] ISO TC97/SC18/WG4/N356/1,2: Message Oricnted Text Interchange Systems, Remote Operation Service (ROS), Part 1: Concepts and Model, Part 2: Basic ROS. January 1986.

[Pappe86] S. Pappe: Anwendung einer Implementierungstcchnik für Kommunikationsprotokolle auf das ISO Kommunikationssteuerungs-Protokoll. Diplomarbcit, Universität Karlsruhe, March 1986.

[Pappe87] S. Pappe, W. Effelsberg, W. Lamersdorf: Database Access in Open Systems. Proc. Networking in Open Systems, Lecture Notes No. 248, Springer Verlag, 1987, pp. 148164.

[West86] C. West: Protocol Validation by Random State Exploration. Proc. 6th Internat. Workshop on Protocol Specification, Testing and Verification, Montreal, Elsevier (1986), pp. 233-242. 